\title{
Metformin: an old drug with potential therapeutic function in antitumor
}

\author{
wei $m u^{1}$, Yunyun Jiang ${ }^{1}$, and Falin $\mathrm{Qu}^{1}$ \\ ${ }^{1}$ Central Military Commission Joint Logistics Support Force 904th Hospital
}

November 8, 2021

\begin{abstract}
Metformin is one of the first-line and widely-used drugs in patients with T2DM due to its safety profile, clinical efficacy and cheap cost. It is clearly that metformin has benefits on lowering hyperglycemia and diabetes-related complications in clinical use. The classic effect of metformin is to reduce hepatic glucose production by inhibiting gluconeogenesis in liver and increase glucose utilization in peripheral tissues. Metformin targets mitochondrial respiratory chain complex I to specifically reduce reactive oxygen species generation to protect cells against oxidative stress-induced cell apoptosis. AMPK complex is a key factor in the action of metformin; however it is inconclusive that whether metformin activate AMPK directly or indirectly. In addition, more and more studies showed that metformin act on gut microbiota to exert anti-hyperglycemia effect. Emerging evidence showed that metformin has off-label function on antitumor therapy; however the underlying mechanism of this property of metformin still remains elusive. Taken together, in this review we provide a new perspective on metformin and repurpose its novel and promising application in antitumor therapy.
\end{abstract}

\section{Metformin: an old drug with potential therapeutic function in antitumor}

Wei Mu*, Yunyun Jiang, Falin Qu*

Department of pharmacy, clinical precision administration center, 904th Hospital of PLA, Wuxi 214000, PR China

\begin{abstract}
Metformin is one of the first-line and widely-used drugs in patients with T2DM due to its safety profile, clinical efficacy and cheap cost. It is clearly that metformin has benefits on lowering hyperglycemia and diabetes-related complications in clinical use. The classic effect of metformin is to reduce hepatic glucose production by inhibiting gluconeogenesis in liver and increase glucose utilization in peripheral tissues. Metformin targets mitochondrial respiratory chain complex I to specifically reduce reactive oxygen species generation to protect cells against oxidative stress-induced cell apoptosis. AMPK complex is a key factor in the action of metformin; however it is inconclusive that whether metformin activate AMPK directly or indirectly. In addition, more and more studies showed that metformin act on gut microbiota to exert antihyperglycemia effect. Emerging evidence showed that metformin has off-label function on antitumor therapy; however the underlying mechanism of this property of metformin still remains elusive. Taken together, in this review we provide a new perspective on metformin and repurpose its novel and promising application in antitumor therapy.
\end{abstract}

Keywords: metformin; hyperglycemia; antitumor; AMPK complex; T2DM

*Corresponding author at: Department of pharmacy, clinical precision administration center, $904^{\text {th }}$

Hospital of PLA, Wuxi 214000, PR China 


\section{E-mail address:germini99@hotmail.com}

\section{1 | INTRODUCTION}

Diabetes is one of chronic metabolic diseases, which become a major public health concern worldwide and causes millions of death every year. According to incomplete statistics, there are almost $10 \%$ of population in the world suffering from diabetics; however $90 \%$ of the total cases were diagnosed with type 2 diabetic mellitus $(\mathrm{T} 2 \mathrm{DM})^{1}$. Over the last 50 years, scientists have made remarkable progress in etiology and therapeutics of diabetic mellitus (DM); one of which is a biguanide agent named metformin that was introduced and then widely used in patients with T2DM in Europe and Canada. Until 1995, metformin was available in the United States $^{2}$. Due to its excellent safety profile, glycemia control in high efficacy, easy to administration and cheap in cost, metformin was received by diabetic patients widely and frequently.

Metformin is one of the first-line anti-hyperglycemia drugs for T2DM treatment with a history of 50 years; and also an effective agent to reduce complications along with diabetics such as vasculopathy, diabetic nephropathy, diabetic foot and infections ${ }^{3}$. Specifically, metformin is safe anti-hyperglycemic agent that does not cause hypoglycemia, even in a high dose concentration. Furthermore, metformin has no significant effects on secretion of hormones such as cortisol, glucagon, somatostatin or growth hormone except glucagon like peptide-1 (GLP-1 $)^{4}$. As a chemical agent with low molecular weight and hydrophilic property, metformin is very suitable for oral preparation and often to be made into enteric-coated tablets.

Currently, it is clear that the effect of metformin is to promote insulin sensitivity and reduce glucose production through inhibiting gluconeogenesis in liver and intestine ${ }^{5}$.In addition, metformin might enhance glucose transporters expression and mediate their translocation onto hepatic cell surface to increase glucose absorption and utilization ${ }^{2}$. It is confirmed the safety and effect of metformin in clinic application; however, more and more reports unraveled new function of metformin such as antitumor, gut microbiota modulation ${ }^{6-8}$, bone homeostasis and anti-aging along with treatment on patients with T2DM. It's amazing that metformin was labeled "nostrum" due to its multifaceted effects; even though there is not enough and strong evidence to support it.

In this Review, we discuss molecular mechanisms and multifaceted effects of metformin in antitumor. In addition, we attempt to figure out the relationship between lowering blood glucose level and antitumor effect of metformin.

\section{| GENERAL MECHANISM AND EFFECT}

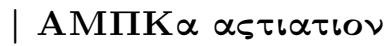

Metformin is the most widely prescribed drug for T2DM treatment in the world until now. It primarily decreases postprandial blood glucose levels via reducing hepatic glucose production and promoting action of insulin in muscle. It is clear that metformin mainly activate AMPK $\alpha$ in the cytoplasm of hepatic cells ${ }^{2}$. Adenosine 5'-monophosphate-activated protein kinase (AMPK) is a heterotrimeric complex with catalytic subunit $\alpha$ and regulatory $\beta$ and $\gamma$ subunits. Metformin phosphorylate AMPK $\alpha$ at site Thr172 independently or via liver kinase B1 (LKB1) activation dependently ${ }^{9}$. Evidently, activation of AMPK $\alpha$ suppress glucose production by downregulating the expression of both gluconeogenesis and lipogenesis enzymes in liver ${ }^{10}$. Likewise, activation of AMPK $\alpha$ increase glucose absorption in liver or muscle by upregulating the expression of glucose transporters $(\mathrm{GLUTs})^{11}$. However activation of AMPK $\alpha$ is not enough to explain anti-hyperglycemia effect of metformin due to AMPK display pleiotropic actions on metabolism.

\section{| Complex I inhibition}

Over two decades study, more evidence on selective inhibition of complex I in respiratory chain by metformin was defined in multiple model organisms and species ${ }^{12,13}$, including cancer cell lines and human primary liver cells $^{14,15}$. Consequently, it leads to increase cellular AMP: ATP ratio and suppress mitochondrial NADH oxidation, all together reduce oxygen consumption rate in cells ${ }^{16}$. In addition, that cells response to this low energy state result in fat acid oxidation acceleration by inhibiting acetyl-CoA carboxylase (ACC) ${ }^{17}$. 
Unfortunately, this action brings about side effect of biguanide drugs, i.e. lactic acidosis, which is the reason for the withdrawal of phenformin. However metformin display high therapeutic effect and safety profile with a lower risk of lactic acidosis. More interestingly, it was reported that metformin has high affinity for copper ions in mitochondrial respiratory chain ${ }^{18}$. It suggested that the interaction between metformin and copper in mitochondrial might contribute to the inhibition of complex I in mitochondrial respiratory chain too.

\section{| Effect on GLP-1 secretion'}

As a chemical drug, metformin display strong hydrophilic property and monovalent cation at physiological $\mathrm{pH}^{19}$. Thus it is difficult for metformin to get through cell membranes without the help from specific transporters. A lot of studies showed that organic cation transporter (OCTs), plasma membrane monoamine transporter (PMAT) and multidrug and toxin extruders (MATEs) are responsible for absorption, distribution and excretion of metabolite in vivo respectively ${ }^{20}$. The actions of metformin in liver and peripheral organs display concentration-dependant and time-dependant manner because it does not combine with serum albumin in circulation system. More emerging evidence indicated that metformin increase blood levels of GLP-1 in patients with T2DM ${ }^{21}$. Moreover, it is reported that metformin has potential effect on enhancing the expression of GLP-1 receptor in pancreatic islet ${ }^{4}$. Other favorable evidence showed that metformin increase intestinal GLP-1 secretion indirectly by sensing the availability of bile acid in patients with T2DM ${ }^{22}$. However, the mechanism of GLP-1 secretion stimulated by metformin remains unclear and elusive.

\section{| ANTITUMOR EFFECT OF METFORMIN}

\section{1 | I/IGF-1-AKT-mTORC1 cascade}

It has reported that metformin had benefit on cancer patients with or without T2DM ${ }^{23}$. Metformin might reduce the blood levels of insulin growth factor-1 (IGF-1), which dampening downstream PI3K/AKT/mTOR signaling pathway that leads to inhibit the proliferation of tumor cells (Fig.1) ${ }^{24}$. In addition, metformin inhibit mTOR complex by AMPK activation directly to suppress proliferation of tumor cells too (Fig.1 $)^{14}$. It is well known that mechanistic target of rapamycin complex (mTOR) includes mTORC1 and mTORC2, which display different functions in metabolism. The primary effect of mTORC1 is to regulate nutrients metabolism and cell growth, thus mTORC1 inhibition results in delaying cell proliferation and nutrients supply. Actually, many oncogenic pathways following mTORC1 mutated frequently in tumor cells that display high activity of $\mathrm{mTORC}^{25}$. Therefore, the possible antitumor mechanism of metformin is to inhibit proliferation of tumor cells by triggering the response of mTOR complex.

\section{2 | AMPK-TET2-5hmC axis}

Another hypothesis to be testified and used to describe antitumor effect of metformin is AMPK-TET2-5hmC axis, which links extracellular hyperglycemia environment to a dynamic regulator 5-hydroxymethylcytosine $(5 \mathrm{hmC}),{ }^{26}$. Tet methyl cytosine dioxygenase2 (TET2), a tumor inhibitor, was destabilized under hyperglycemia and lost the function of $5 \mathrm{hmC}$ regulation, which is a pivotal epigenetic change in tumorgenesis $^{27}$. That activation of TET2 increase levels of $5 \mathrm{hmC}$ of histone and inhibit hydroxymethlome and transcriptome ${ }^{28,29}$. Notably, AMPK is an upstream regulator of TET2; thereby activation of AMPK leads to TET2 phosphorylation and stabilization ${ }^{26,27}$. As a result, TET2 looks like a molecular switch to control and coordinate $5 \mathrm{hmC}$ levels, which process can be regulated by AMPK activation that is mediated by metformin (Fig.1 $)^{30}$. AMPK-TET2-5hmC pathway is new illumination on the antitumor effect of metformin independently of classic mTORC1 pathway ${ }^{26}$. This regulation in epigenetic may provide a new clue on cancer prevention and clinical research.

\section{3 | Clinic evidence of metformin}

In clinical research, there are a lot of exciting reports and investigations about metformin's potential antitumor effect. Data from epidemiological support the evidence that metformin reduce the mortality of cancerrelated diseases and risk of tumorgenesis ${ }^{31}$. In a clinical randomized study, that metformin use in diabetic patients with non-small cell lung cancer was associated with a better overall survival (OS) independently ${ }^{32}$. In addition, that combination use of epidermal growth factor receptor-tyrosine kinase inhibitor (EGFR-TKI) 
with metformin improved OS for non-diabetic patients with advanced lung adenocarcinoma more than that of EGFR-TKIs alone ${ }^{32}$.Another report revealed that more than 2 years of metformin use reduce the risk of cervical cancer significantly ${ }^{33}$.It is clear that high levels of $4 \mathrm{E}$-binding proteins (4E-BPs) expression in tumor cells may ameliorate clinical chemotherapy. A case indicated that metformin activated 4E-BPs to induce apoptosis in hepatocellular carcinoma (HCC $)^{34}$. Another case showed that metformin could induce apoptosis in HCC via AMPK/p53/miR-23a/FOXA1 signaling cascade ${ }^{35}$. It is reported that sunitinib combined with metformin use benefits survival rate in metastatic renal cell carcinoma (mRCC) patients ${ }^{36,37}$. Furthermore, metformin mediate high expression of miR-26a effectively to reduce the production of phosphatase and tensin homologue deleted on chromosome 10 (PTEN) and enhancer of zeste homolog2 (EHZ2), both of which are key regulator in tumorgenesis, to increase apoptosis of breast cancer cell ${ }^{38}$. A systemic review suggested that metformin had the greatest adjuvant benefits on patients with colorectal and prostate cancer $^{39}$, significantly for those who received radical radiotherapy ${ }^{40}$. It is reported that metformin blocked programmed cell death 1 ligand (PD-L1) and programmed cell death 1 (PD-1) binding to promote antitumor cytotoxic T lymphocytes (CTL) immunity by activating AMPK signaling cascade to phosphorylate PD-L1 on site 195. It leaded to PD-L1 abnormal glycosylation and PD-L1 degradation by ER-associated degradation (ERAD) subsequently ${ }^{41}$. In addition, metformin attenuate the function of cancer stem cells (CSCs) to alleviate therapeutic resistance such as chemotherapy and radiotherapy in prostate, gastrointestinal and pancreatic cancer ${ }^{42,43}$. Likewise, metformin selectively inhibit intercellular chloride channel-1 to suppress proliferation of CSCs in human glioblastoma ${ }^{44}$.

\section{4 | CONCLUSION AND PERSPECTIVE}

The drug metformin originates from plant extracts of goat's rue or French lilac ${ }^{2}$. It was used as first line anti-hyperglycemia drugs in clinic for more than half century with targets primarily on gut and liver to exert effect on lowering blood glucose level. In molecular mechanism, metformin activate AMPK directly or via LKB1 in hepatocytes or intestinal cells ${ }^{45}$. Previous studies suggested that activation of LKB1-AMPK signal cascade suppressed relevant gluconeogenesis gene transcription through dephosphorylation and sequestration of CREB regulated transcription coactivator2 (CRTC2) in cytoplasm ${ }^{46,47}$. However, this point of view was challenged by other studies that metformin did not inhibit gene expression but activity of enzyme in gluconeogenesis in liver; because metformin could repress glucose production and gene expression of gluconeogenesis in mice with hepatic LKB1 or AMPK catalytic subunits deficient, which suggested that metformin inhibited gluconeogenesis in hepatocytes independent of AMPK activation or LKB1 regulation ${ }^{48,49}$. Furthermore, other studies revealed that metformin inactivated CRTC2 independently of AMPK activation to inhibit gluconeogenesis ${ }^{50}$. In addition, it was reported that metformin reduced blood glucose levels in hepatic AMPK-deficient mice without affecting pyruvate tolerance and normal glycemia ${ }^{48}$.

Early studies showed that metformin acted on mitochondria directly ${ }^{51}$; however the exact mechanism that acting on respiratory chain complex I in mitochondrial has been reported by two independent research teams until $2000^{52,53}$. Comparing with classical potent uncharged hydrophobic inhibitors of mitochondrial respiratory chain complex I such as piericidin A and rotenone ${ }^{54}$, the inhibitive effect of metformin on mitochondrial respiratory chain complex I is reversible and weak, which cause a mild reduction in ATP generation and moderate rise in the ratio of AMP: ATP in liver cells ${ }^{53}$. Previous studies indicated that metformin distributed mainly within the cytoplasm in hepatocytes ${ }^{55}$; however new evidence demonstrated that metformin mainly concentrated on mitochondrial membrane ${ }^{56}$. Besides, it is still inconclusive whether metformin inhibits respiratory chain complex I in mitochondria directly or not ${ }^{57}$. On pharmacokinetics (PK) and pharmacodynamics (PD) study, a large-scale meta-analysis showed that there was no significance in statistics of the polymorphisms in transporters involved in PKs when glycemia responding to metformin ${ }^{20}$. However, there is close associations between variations on Glut2 gene and glycemia response to metformin in PD genetics study $^{9}$. Accordingly, the whole picture of metformin acting on mitochondrial respiratory chain complex I is still blurred owing to multiple factors influence.

In tumor cells, it is clear that AMPK is dampened while mTORC1 is extraordinary active ${ }^{49}$. Activation of AMPK lead to p53 phosphorylation to induce tumor cell apoptosis and inhibit fatty acid synthesis to 
regulate tumor cell growth by reducing the material source for assembling tumor cell membranes (Fig.1 ${ }^{17}$. Furthermore, activation of AMPK increased deacetylation and methylation of histone to regulate anti-tumor gene expression negative ${ }^{58}$. In System, metformin downregulate insulin/insulin growth factor1 (I/IGF-1) signaling cascade to inhibit tumor development, repress nuclear factor- $x \mathrm{~B}(\mathrm{NF}-\varkappa \mathrm{B})$ signaling pathway to reduce proinflammatory cytokines release and cell adhesion molecules, promote tumor cells to release lactate to suppress Warburg effect (Fig.1 $)^{59}$. It is reported that diabetic patients with lower risk of tumor after metformin treatment, but whether it benefit on non-diabetic patients or not? To our surprise, there are some encouraging and promising results from clinical trials on non-diabetic cancer patients with metformin treatment ${ }^{9}$. Scientists have initiated a lot of clinical trials about metformin around the world, most of which were in Asia-Pacific, Europe Union and North America (Fig.2). Admittedly, cancer is not single disease; actually, it's a kind of systemic lesions that displays multiple behaviors in phenotypes and polymorphism in genotypes. Until now, scientists can't reach agreement on the antineoplastic effect of metformin owing to multi-diversity property of tumor cells and multifaceted actions of metformin. Thus, it will be a challenging and exciting work to illuminate antitumor effect of metformin in future.

In conclusion, as far as we know, the exact mechanism of metformin is still confusing owing to its potential therapeutic effects on various diseases. The new effect of metformin on GLP-1 secretion and gut microbiota challenged the classic model of liver-centered action mechanism ${ }^{4,21}$. However, we should reevaluate research itself with respect to its diversity. First, most of results from in vitro or preclinical studies depended on the use of metformin in high concentration that is far exceeding the clinic use in therapy. Notably, the absolute bioavailability of metformin is around $50 \%-60 \%$ that is lower than other anti-hyperglycemia drugs ${ }^{58}$. So, it's not a good option for patients to increase dose of metformin because of side effects such as kidney impairment and lactic acidosis. To our dismay, there is little report on the normal concentration use of metformin out of clinic. If we want to rise up local concentration in specific tissue for treatment, we have to rely on new drug delivery system such as microparticles or nanoparticles to deliver metformin into target tissue to exert therapeutic effect ${ }^{60,61}$. Second, the antitumor effect of metformin is so complicated that there is no general mechanism to explain it clearly. Therefore, it brings about more uncertainty of metformin in cancer treatment. Furthermore, both the polymorphisms of gene in drug metabolism and multi-diversity of cancer genome are barriers in achieving consistent evaluation of metformin in clinical trials. Last but not the least; we should delve into the relationship between antitumor effect of metformin and its primary effect on lowering blood glucose level. Owing to its benefit of no hypoglycemia, metformin is friendly for non-diabetic patients in antitumor treatment. Finally, metformin will become a promising and encouraging drug in future.

\section{ACKNOWLEDGEMENTS}

This work was supported by grants from the National Basic Research Program (973 Project) of China (No. 2013CB530802) and the National Natural Science Foundation of China (No. 81471396, 81470425 and 81681260239). Thanks Dr. Haellman for providing templates for Figures 1-4.

\section{COMPETING INTEREST}

There are no competing interests to declare.

\section{CONTRIBUTORS}

The ${ }^{*}$ authors contributed equally to all aspects of the article.

\section{ORCID}

Wei Mu https://orcid.org/000-0002-1894-9172

\section{Reference}

1. Schwarz PE, Gallein G, Ebermann D, et al. Global Diabetes Survey-anannual report on quality of diabetes care. Diabetes Res Clin Pract . Apr 2013;100(1):11-8. doi:10.1016/j.diabres.2012.11.008 
2. Bailey CJ, Turner RC. Metformin. $\quad N$ Engl $J$ Med . Feb 291996;334(9):574-9. doi:10.1056/NEJM199602293340906

3. Scarpello JH, Howlett HC. Metformin therapy and clinical uses. Diab Vasc Dis Res. Sep 2008;5(3):15767. doi:10.3132/dvdr.2008.027

4. Bahne E, Sun EWL, Young RL, et al. Metformin-induced glucagon-like peptide-1 secretion contributes to the actions of metformin in type 2 diabetes. JCI Insight. Dec2018;3(23)doi:10.1172/jci.insight.93936

5. Hunter RW, Hughey CC, Lantier L, et al. Metformin reduces liver glucose production by inhibition of fructose-1-6-bisphosphatase. Nat Med. Sep 2018;24(9):1395-1406. doi:10.1038/s41591-018-0159-7

6. Forslund K, Hildebrand F, Nielsen $\mathrm{T}$, et al. Disentangling type 2 diabetes and metformin treatment signatures in the human gut microbiota. Nature. Dec 10 2015;528(7581):262-266. doi:10.1038/nature15766

7. Libby G, Donnelly LA, Donnan PT, Alessi DR, Morris AD, Evans JM. New users of metformin are at low risk of incident cancer: a cohort study among people with type 2 diabetes. Diabetes Care. Sep 2009;32(9):1620-5. doi:10.2337/dc08-2175

8. Pernicova I, Korbonits M. Metformin-mode of action and clinical implications for diabetes and cancer. Nat Rev Endocrinol. Mar 2014;10(3):143-56. doi:10.1038/nrendo.2013.256

9. Zhou Z, Tang Y, Jin X, et al. Metformin Inhibits Advanced Glycation End Products-Induced Inflammatory Response in Murine Macrophages Partly through AMPK Activation and RAGE/NFkappaB Pathway Suppression. J Diabetes Res. 2016;2016:4847812. doi:10.1155/2016/4847812

10. Madiraju AK, Erion DM, Rahimi Y, et al. Metformin suppresses gluconeogenesis by inhibiting mitochondrial glycerophosphate dehydrogenase. Nature. Jun 26 2014;510(7506):542-6. doi:10.1038/nature13270

11. Zheng D, MacLean PS, Pohnert SC, et al. Regulation of muscle GLUT-4 transcription by AMP-activated protein kinase. J Appl Physiol (1985). Sep 2001;91(3):1073-83. doi:10.1152/jappl.2001.91.3.1073

12. Batandier C, Guigas B, Detaille D, et al. The ROS production induced by a reverse-electron flux at respiratory-chain complex 1 is hampered by metformin. J Bioenerg Biomembr. Feb 2006;38(1):33-42. doi:10.1007/s10863-006-9003-8

13. Cameron AR, Logie L, Patel K, et al. Metformin selectively targets redox control of complex I energy transduction. Redox Biol. Apr 2018;14:187-197. doi:10.1016/j.redox.2017.08.018

14. Heckman-Stoddard BM, DeCensi A, Sahasrabuddhe VV, Ford LG. Repurposing metformin for the prevention of cancer and cancer recurrence. Diabetologia. Sep 2017;60(9):1639-1647. doi:10.1007/s00125017-4372-6

15. Kurelac I, Iommarini L, Vatrinet R, et al. Inducing cancer indolence by targeting mitochondrial Complex I is potentiated by blocking macrophage-mediated adaptive responses. Nat Commun. Feb 22 2019;10(1):903. doi:10.1038/s41467-019-08839-1

16. Vial G, Detaille D, Guigas B. Role of Mitochondria in the Mechanism(s) of Action of Metformin. Front Endocrinol (Lausanne). 2019;10:294. doi:10.3389/fendo.2019.00294

17. Fullerton MD, Galic S, Marcinko K, et al. Single phosphorylation sites in Acc1 and Acc2 regulate lipid homeostasis and the insulin-sensitizing effects of metformin. Nat Med. Dec 2013;19(12):1649-54. doi:10.1038/nm.3372

18. Logie L, Harthill J, Patel K, et al. Cellular responses to the metal-binding properties of metformin. Diabetes. Jun 2012;61(6):1423-33. doi:10.2337/db11-0961

19. Hardie DG. AMPK-sensing energy while talking to other signaling pathways. Cell Metab. Dec 2 2014;20(6):939-52. doi:10.1016/j.cmet.2014.09.013

20. Dujic T, Zhou K, Yee SW, et al. Variants in Pharmacokinetic Transporters and Glycemic Response to Metformin: A Metgen Meta-Analysis. Clin Pharmacol Ther. Jun 2017;101(6):763-772. doi:10.1002/cpt.567

21. DeFronzo RA, Buse JB, Kim T, et al. Once-daily delayed-release metformin lowers plasma glucose and enhances fasting and postprandial GLP-1 and PYY: results from two randomised trials. Diabetologia. Aug 2016;59(8):1645-54. doi:10.1007/s00125-016-3992-6 
22. Bronden A, Alber A, Rohde U, et al. Single-Dose Metformin Enhances Bile Acid-Induced GlucagonLike Peptide-1 Secretion in Patients With Type 2 Diabetes. J Clin Endocrinol Metab. Nov 1 2017;102(11):4153-4162. doi:10.1210/jc.2017-01091

23. Decensi A, Puntoni M, Goodwin P, et al. Metformin and cancer risk in diabetic patients: a systematic review and meta-analysis. Cancer Prev Res (Phila). Nov 2010;3(11):1451-61. doi:10.1158/19406207.CAPR-10-0157

24. Zi F, Zi H, Li Y, He J, Shi Q, Cai Z. Metformin and cancer: An existing drug for cancer prevention and therapy. Oncol Lett. Jan 2018;15(1):683-690. doi:10.3892/ol.2017.7412

25. Saxton RA, Sabatini DM. mTOR Signaling in Growth, Metabolism, and Disease. Cell. Apr 6 2017;169(2):361-371. doi:10.1016/j.cell.2017.03.035

26. Wu D, Hu D, Chen H, et al. Glucose-regulated phosphorylation of TET2 by AMPK reveals a pathway linking diabetes to cancer. Nature. Jul 2018;559(7715):637-641. doi:10.1038/s41586-018-0350-5

27. Wang Y, Zhang Y. Regulation of TET protein stability by calpains. Cell Rep. Jan 30 2014;6(2):278-84. doi:10.1016/j.celrep.2013.12.031

28. Williams K, Christensen J, Pedersen MT, et al. TET1 and hydroxymethylcytosine in transcription and DNA methylation fidelity. Nature. May 19 2011;473(7347):343-8. doi:10.1038/nature10066

29. $\mathrm{Xu} \mathrm{Y}, \mathrm{Wu} \mathrm{F}$, Tan L, et al. Genome-wide regulation of $5 \mathrm{hmC}, 5 \mathrm{mC}$, and gene expression by Tet1 hydroxylase in mouse embryonic stem cells. Mol Cell. May 20 2011;42(4):451-64. doi:10.1016/j.molcel.2011.04.005

30. Ko M, Huang Y, Jankowska AM, et al. Impaired hydroxylation of 5-methylcytosine in myeloid cancers with mutant TET2. Nature. Dec 9 2010;468(7325):839-43. doi:10.1038/nature09586

31. Gandini S, Puntoni M, Heckman-Stoddard BM, et al. Metformin and cancer risk and mortality: a systematic review and meta-analysis taking into account biases and confounders. Cancer Prev Res (Phila). Sep 2014;7(9):867-85. doi:10.1158/1940-6207.CAPR-13-0424

32. Arrieta O, Barron F, Padilla MS, et al. Effect of Metformin Plus Tyrosine Kinase Inhibitors Compared With Tyrosine Kinase Inhibitors Alone in Patients With Epidermal Growth Factor ReceptorMutated Lung Adenocarcinoma: A Phase 2 Randomized Clinical Trial. JAMA Oncol. Nov 1 2019;5(11):e192553. doi:10.1001/jamaoncol.2019.2553

33. Tseng CH. Metformin use and cervical cancer risk in female patients with type 2 diabetes. Oncotarget. Sep 13 2016;7(37):59548-59555. doi:10.18632/oncotarget.10934

34. Bhat M, Yanagiya A, Graber T, et al. Metformin requires 4E-BPs to induce apoptosis and repress translation of Mcl-1 in hepatocellular carcinoma cells. Oncotarget. Aug 1 2017;8(31):50542-50556. doi:10.18632/oncotarget.10671

35. Sun Y, Tao C, Huang X, et al. Metformin induces apoptosis of human hepatocellular carcinoma HepG2 cells by activating an AMPK/p53/miR-23a/FOXA1 pathway. Onco Targets Ther. 2016;9:2845-53. doi:10.2147/OTT.S99770

36. Becker C, Jick SS, Meier CR, Bodmer M. Metformin and the risk of renal cell carcinoma: a case-control analysis. Eur J Cancer Prev. May 2017;26(3):257-262. doi:10.1097/CEJ.0000000000000246

37. Hamieh L, McKay RR, Lin X, Moreira RB, Simantov R, Choueiri TK. Effect of Metformin Use on Survival Outcomes in Patients With Metastatic Renal Cell Carcinoma. Clin Genitourin Cancer. Apr 2017;15(2):221-229. doi:10.1016/j.clgc.2016.06.017

38. Cabello P, Pineda B, Tormo E, Lluch A, Eroles P. The Antitumor Effect of Metformin Is Mediated by miR-26a in Breast Cancer. Int J Mol Sci. Aug 10 2016;17(8)doi:10.3390/ijms17081298

39. Coyle C, Cafferty FH, Vale C, Langley RE. Metformin as an adjuvant treatment for cancer: a systematic review and meta-analysis. Ann Oncol. Dec 2016;27(12):2184-2195. doi:10.1093/annonc/mdw410

40. Zannella VE, Dal Pra A, Muaddi H, et al. Reprogramming metabolism with metformin improves tumor oxygenation and radiotherapy response. Clin Cancer Res. Dec 15 2013;19(24):6741-50. doi:10.1158/1078-0432.CCR-13-1787

41. Cha JH, Yang WH, Xia W, et al. Metformin Promotes Antitumor Immunity via EndoplasmicReticulum-Associated Degradation of PD-L1. Mol Cell. Aug 16 2018;71(4):606-620 e7. doi:10.1016/j.molcel.2018.07.030 
42. Di Francesco AM, Toesca A, Cenciarelli C, Giordano A, Gasbarrini A, Puglisi MA. Metabolic Modification in Gastrointestinal Cancer Stem Cells: Characteristics and Therapeutic Approaches. J Cell Physiol. Oct 2016;231(10):2081-7. doi:10.1002/jcp.25318

43. Mayer MJ, Klotz LH, Venkateswaran V. Metformin and prostate cancer stem cells: a novel therapeutic target. Prostate Cancer Prostatic Dis. Dec 2015;18(4):303-9. doi:10.1038/pcan.2015.35

44. Saini N, Yang X. Metformin as an anti-cancer agent: actions and mechanisms targeting cancer stem cells. Acta Biochim Biophys Sin (Shanghai). Feb 1 2018;50(2):133-143. doi:10.1093/abbs/gmx106

45. Nakada D, Saunders TL, Morrison SJ. Lkb1 regulates cell cycle and energy metabolism in haematopoietic stem cells. Nature. Dec 2 2010;468(7324):653-8. doi:10.1038/nature09571

46. Miller RA, Chu Q, Xie J, Foretz M, Viollet B, Birnbaum MJ. Biguanides suppress hepatic glucagon signalling by decreasing production of cyclic AMP. Nature. Feb 14 2013;494(7436):256-60. doi:10.1038/nature11808

47. Shaw RJ, Lamia KA, Vasquez D, et al. The kinase LKB1 mediates glucose homeostasis in liver and therapeutic effects of metformin. Science. Dec 9 2005;310(5754):1642-6. doi:10.1126/science.1120781

48. Foretz M, Hebrard S, Leclerc J, et al. Metformin inhibits hepatic gluconeogenesis in mice independently of the LKB1/AMPK pathway via a decrease in hepatic energy state. J Clin Invest. Jul 2010;120(7):2355-69. doi:10.1172/JCI40671

49. Howell JJ, Hellberg K, Turner M, et al. Metformin Inhibits Hepatic mTORC1 Signaling via DoseDependent Mechanisms Involving AMPK and the TSC Complex. Cell Metab. Feb 7 2017;25(2):463471. doi:10.1016/j.cmet.2016.12.009

50. He L, Sabet A, Djedjos S, et al. Metformin and insulin suppress hepatic gluconeogenesis through phosphorylation of CREB binding protein. Cell. May 15 2009;137(4):635-46. doi:10.1016/j.cell.2009.03.016

51. Schafer G. Site-specific uncoupling and inhibition of oxidative phosphorylation by biguanides. II. Biochim Biophys Acta. Feb 25 1969;172(2):334-7. doi:10.1016/0005-2728(69)90077-2

52. El-Mir MY, Nogueira V, Fontaine E, Averet N, Rigoulet M, Leverve X. Dimethylbiguanide inhibits cell respiration via an indirect effect targeted on the respiratory chain complex I. J Biol Chem. Jan 7 2000;275(1):223-8. doi:10.1074/jbc.275.1.223

53. Owen MR, Doran E, Halestrap AP. Evidence that metformin exerts its anti-diabetic effects through inhibition of complex 1 of the mitochondrial respiratory chain. Biochem J. Jun 15 2000;348 Pt 3:607-14.

54. Degli Esposti M. Inhibitors of NADH-ubiquinone reductase: an overview. Biochim Biophys Acta. May 6 1998;1364(2):222-35. doi:10.1016/s0005-2728(98)00029-2

55. Wilcock C, Wyre ND, Bailey CJ. Subcellular distribution of metformin in rat liver. J Pharm Pharmacol. Jun 1991;43(6):442-4. doi:10.1111/j.2042-7158.1991.tb03507.x

56. Bridges HR, Jones AJ, Pollak MN, Hirst J. Effects of metformin and other biguanides on oxidative phosphorylation in mitochondria. Biochem J. Sep 15 2014;462(3):475-87. doi:10.1042/BJ20140620

57. Fontaine E. Metformin-Induced Mitochondrial Complex I Inhibition: Facts, Uncertainties, and Consequences. Front Endocrinol (Lausanne). 2018;9:753. doi:10.3389/fendo.2018.00753

58. Graham GG, Punt J, Arora M, et al. Clinical pharmacokinetics of metformin. Clin Pharmacokinet. Feb 2011;50(2):81-98. doi:10.2165/11534750-000000000-00000

59. Aatsinki SM, Buler M, Salomaki H, Koulu M, Pavek P, Hakkola J. Metformin induces PGC-1alpha expression and selectively affects hepatic PGC-1alpha functions. Br J Pharmacol. May 2014;171(9):235163. doi:10.1111/bph.12585

60. Maruthur NM, Tseng E, Hutfless S, et al. Diabetes Medications as Monotherapy or Metformin-Based Combination Therapy for Type 2 Diabetes: A Systematic Review and Meta-analysis. Ann Intern Med. Jun 7 2016;164(11):740-51. doi:10.7326/M15-2650

61. Fujita Y, Inagaki N. Metformin: New Preparations and Nonglycemic Benefits. Curr Diab Rep. Jan 2017;17(1):5. doi:10.1007/s11892-017-0829-8

62. Mirazi N, Shoaei J, Khazaei A, Hosseini A. A comparative study on effect of metformin and metforminconjugated nanotubes on blood glucose homeostasis in diabetic rats. Eur J Drug Metab Pharmacokinet. Sep 2015;40(3):343-8. doi:10.1007/s13318-014-0213-x 

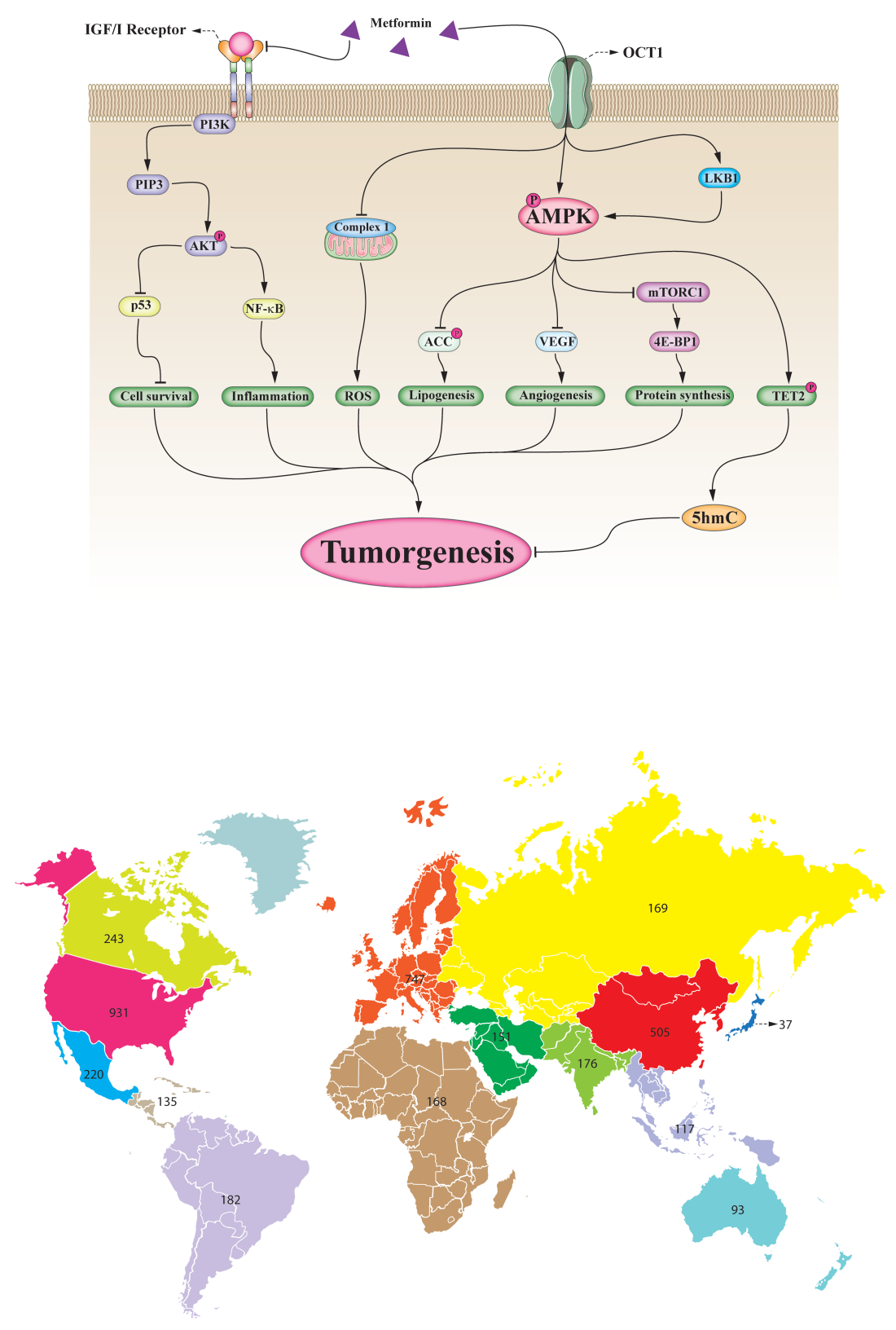